\title{
Editorial
}

\section{Challenges Ahead for Advanced Training Programs in Pediatric Dentistry in the Area of Sedation for Pediatric Dentists and the Commission on Dental Accreditation}

\author{
John E. Nathan, DDS, MDS* \\ Department of Pediatric Dentistry, University of Alabama, Birmingham, and Case Western Reserve University, Cleveland, USA \\ *Corresponding author \\ John E. Nathan, DDS, MDS \\ Adjunct Professor, Department of Pediatric Dentistry, University of Alabama, Birmingham, and Case Western Reserve University, Cleveland, USA; \\ E-mail: jnathandds@gmail.com
}

\section{Article information}

Received: November 3 $3^{\text {rd }}, 2019$; Accepted: November 8 ${ }^{\text {th }}$, 2019; Published: November 15 ${ }^{\text {th }}, 2019$

\section{Cite this article}

Nathan JE. Challenges ahead for advanced training programs in pediatric dentistry in the area of sedation for pediatric dentists and the commission on dental accreditation. Pediatr Neonatal Nurs Open J. 2019; 6(I): e8-e9. doi: 10.17140/PNNOJ-6-e0I0

\begin{abstract}
mongst the challenges faced by advanced training programs 1 in pediatric dentistry is adequate preparation of its students or residents to make use of the most effective and safe sedative agents and combinations for managing the diverse range of noncoping behaviors of pre-cooperative and non-cooperative pediatric patients. The dilemma is complicated by a number of factors. Foremost is a constricted interval of time in which to acquire sufficient exposure and expertise in both didactic and clinical applications. Having sufficient patient populations reflecting various levels of patient apprehension, resistance, and dental pathology has potential to limit student and resident experience is another complication. Faculty variability with respect to expertise, comfort level, and firsthand experience making use of a less than diverse arsenal of agents no doubt has potential impact on resident experience. This is particularly disconcerting where programs have elected to reduce their armamentariums to the extent that experience is gained exclusively with use of a single medication.
\end{abstract}

Fulfillment of accreditation standards in this area is recognizably a challenge for some if not many programs. Didactic training must include sufficient exposure and knowledge of both classical and contemporary literature that provides clinical opinion, substantive retrospective and prospective evidence-based support of the efficacy, safety and recovery parameters for numerous agents for successful management of patient anxiety and resistive behaviors. To ensure safety across all levels of sedation, students must be able to demonstrate competence in airway management and recognition of developing adverse physiologic responses. Knowledge and proficiency in physical diagnosis and pharmacology of sedative and analgesic medication are prerequisite skills to demonstrate competency. Lastly, clinical exposure must be as diverse as possible using a broad range of agents and dosing appro- priate for the specific needs of a given patient.

Considerable energies have been expended by the Commission on Dental Accreditation (CODA) to determine what constitutes minimally acceptable (not necessarily optimal) experiences in the area of pediatric sedation training. Factors which influence the commission's recommendations and required expectations for faculty competence are in some respects limited, but nevertheless carry foremost the responsibility to protect the public. A critical and comprehensive review of the existing pediatric sedation literature from the perspective of pediatricians, anesthesiologists, oral and maxillofacial surgeons and pediatric dental specialists reveals a paucity of controlled investigations to clarify let alone shed considerable light on what agents have proven track records of efficacy and safety. Despite the existence and perpetual revision of existing national safety guidelines for the use of pediatric sedation, mishaps involving morbidity and catastrophic outcome continue to be appear. Nevertheless, while it is the global intent of regulatory agencies to protect children, parents and dentists from such occurrences, resources and manpower are not readily in place to guarantee provider compliance on either a state to state or national level. Compliance and safety concerns however within training programs falls under the auspices of the program director and faculty in attendance. Within private practice, providers and their staffs must satisfy state regulations and guidelines for safety.

For discussion purposes, the CODA has outlined specific minimum experiences that must take place within a twenty-fourmonth training program. Beyond the use of nitrous oxide alone, students/residents must experience a minimum of 50 sedation experiences, half of which must occur as primary operator. No determination or mention to date exists to identify what agents must 
be used within these experiences. All decisions as to what agents are employed are made by the respective program directors and their institutional formulary service. In some states, specific agents are excluded due to an occurrence of adverse reactions, miss-use or abuse, based on litigation outcomes and not necessarily science. Under these circumstances, decisions on what to include or exclude fall outside the realm of program directors. This likely contributes to a reticence for CODA to identify what agents must be taught in training programs. This particular judgment, however, is subject to significant concern. Surveys both recent and of old have identified use of a wide range of agents at but a few institutions; others make use only of agents which possess reversal capabilities. If optimal agents were readily known and this was a perfect world, these types of limitations might be considered reasonable. From the perspective of this author, the latter approach represents a major deficiency in preparing residents for the needs of challenging children. For some institutions, for all intent and purposes, large numbers of patients who could conceivably be treated safely with conscious sedation are being channeled to general anesthesia to transfer all risk to anesthesiologists. Whether or not this falls within the best interests of these children depends on the judgment of the parent and provider. Nevertheless, the CODA has systematically outlined criteria in conjunction with and consistent with the American Academy of Pediatric Dentistry (AAPD) for which sedative management of challenging behaviors maintaining consciousness is considered appropriate. It is noteworthy that sedation continuing education courses offered by the AAPD while alluding to the use of some regimens, avoid recommendations of what constitute viable agents. In an otherwise ideal world, clarification of a range of agents for which students and residents should receive instruction and experience would be helpful to mitigate this deficiency. Without substantive data, however, to demonstrate evidence-based support for efficacy and safety, it is not surprising that these agencies to date refrain from their inclusion. Without consistent levels of comfort of program directors using a broad regimen of agents, student exposure/preparation can be expected to be insufficient.

One conceptual platform remains. Complete reliance on agents such as midazolam, with or without nitrous oxide, remains an unfortunate state of affairs for our management arsenals for children and advanced training programs. Extensive study of midazolam has been performed. Distinct limitations with respect to efficacy and duration of action render it of limited value for anything but short and ultra-short duration visits. Where anxiety levels and resistance are severe, virtually all studies report the need for persistent adjunctive physical restraint to complete treatment. Perhaps future reports will enable better judgments associated with proper dosing limits for various agents and situations.

\section{DISCLAIMER}

This editorial reflects the opinion of the author and in no way should be construed to represent the views of the American Academy of Pediatric Dentistry (AAPD) or Commission on Dental Accreditation (CODA). 